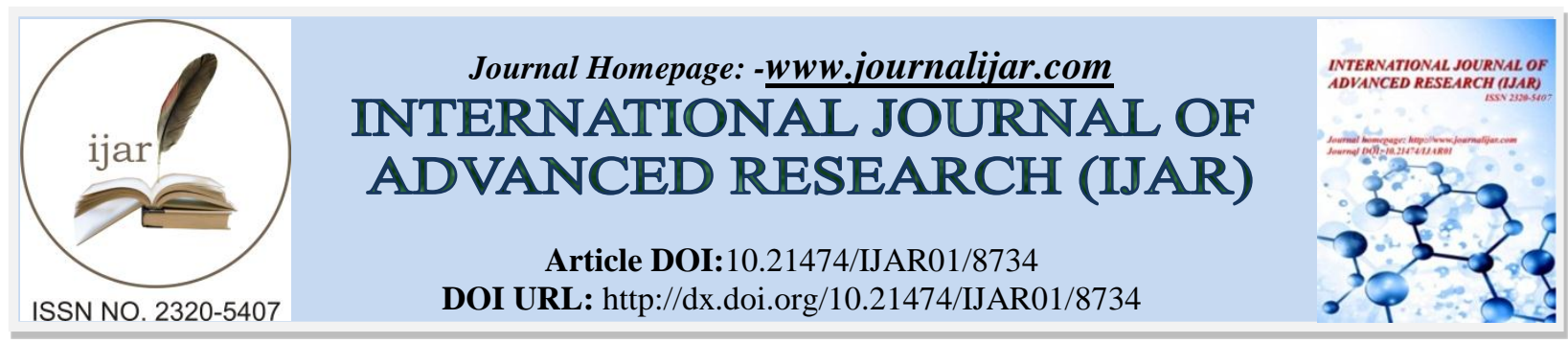

RESEARCH ARTICLE

\title{
A STUDY OF HUMAN RENAL PELVICALYCEAL SYSTEM IN RELATION TO THE BRANCHES OF RENAL ARTERIES.
}

\author{
Dr Sama Abdulkadar and Dr Arivu Selvan. \\ Department of Anatomy, GMC Kozhikode.
}

\section{Manuscript Info}

\section{Manuscript History}

Received: 18 January 2019

Final Accepted: 20 February 2019

Published: March 2019

\begin{abstract}
Introduction: A thorough anatomical knowledge on the patterns of pelvicalyceal system and its relation to the renal segmental arteries is of paramount importance in endourological procedures.

Aim: To study the variations in the pattern of arrangement of major calices, segmental arteries and their relation to each other.

Method: 100 dissection specimens (70 adult, 30 foetuses) and 50 endocasts were studied at a tertiary center in northern Kerala.

Results: The major calyces were arranged in three patterns, tricaliceal (Adults- 44.17\%; Foetus-30\%), bicaliceal (Adults- 24.17\%; Foetus26.67\%), multicaliceal (Adults- 25.83\%; Foetus- 40\%) and rest belonging to none of the above. The primary division of renal artery (RA) was anterior-posterior (92\%) and upper-lower (8\%). Anterior division showed a preponderance of type (TY) 2 among the 8 types. Apical segmental artery (SA) was divided into 6 types, with 50\% showing TY1 pattern. $62 \%$ showed TY1 of upper SA (among 3 types) and $84 \%$ of TY 1 of lower SA (total-4 types). Middle SA showed 54\% TY1 among 4 types. Posterior SA divided in magistral (62\%), bifurcating (30\%), cruciate ( $8 \%$ ) patterns. Superior calyces were related to anterior and posterior branches in $88 \%$, anterior alone in $10 \%$ and posterior alone in 2\%; mid-zone to anterior division only; inferior pole to branches of lower SA. Dorsal kidney was related to posterior SA which was related to upper infundibulum $(60 \%)$ or crossed middle posterior surface of pelvis (40\%).

Conclusion: Numerous variations in patterns of calyces, SAs and their relation to each other were observed, which could not be discerned from studying the external surface of the kidney. This emphasizes the importance of pre-operative angiography and IVU prior to any endourological intervention.
\end{abstract}

Copy Right, IJAR, 2019,. All rights reserved.

\section{Introduction:-}

Collecting system of the kidney comprises of collecting tubules which join together to form collecting ducts. These ducts open at the pyramids in to the minor calyces which unite together to form two to three major calyces. These in turn join to form, the renal pelvis. The variations in the gross structure of the renal collecting system are probably as numerous as there are individuals and thus can be liked to fingerprints. The bilateral collecting systems present in 
any single individual are often similar but are rarely identical and not uncommonly, may be quite different even from one another ${ }^{1}$.

Advances in surgical techniques are based most firmly on precise researches into the structure and function of tissue concerned $^{2}$. Knowledge of calyceal anatomy is helpful for the better understanding and interpretation of standard Intravenous urography (IVU) ${ }^{1}$. Great technological advances due to modern diagnostic imaging techniques, particularly in the field of urology, in non-interventionist radiological procedures and in the area of surgery, have emphasized having better morphological referents for the renal vascularization pattern. Even though imaging techniques have good resolution, direct anatomical studies lead to recognizing anatomical vascularization patterns in greater detail, offering a referent having great usefulness for interpreting, managing, and surgical approaches and diagnosing functional alterations ${ }^{3}$.

The importance of being familiar with the renal artery variability, which has become indispensable to urological surgery, has increased as a result of the large number of renal transplants and vascular reconstructions ${ }^{4}$. Segmental artery ligation reduces the risk for renal parenchyma during laparoscopic partial nephrectomies to a minimum ${ }^{5}$.

A serious complication of percutaneous manipulation and intrarenal operations is injury to a major blood vessel ${ }^{6}$. To avoid such injury one must know the position of the renal artery and its branches in relation to the renal pelvis and calices. Excretory urograms (IVPs) show the collecting system only in 1 plane and do not show the arterial system. Thus it is extremely difficult to imagine these intrarenal structures and their relationship in three dimensions as they are in reality ${ }^{7}$. Hence a study of the relationship between the two using endocasts will be of immense value to urologists.

The present study thus aims at describing the variations and relationship of the pelvicalyceal system with the renal segmental arteries.

\section{Materials and methods:-}

This is a descriptive study conducted in the departments of anatomy and forensic medicine in a tertiary center. Exclusion criteria included kidneys with external injuries and those cases were death due to poisoning could not be ruled out. 150 kidneys were studied, 100 by dissection and 50 by casting. The dissected specimens included 70 adult and 30 foetal specimens. Foetal age varied from 16 weeks to term, gestational age calculated from the crown rump length. The kidneys were dissected out as described in Cunningham's manual of Dissection, Vol.3. The shape, position of the hilum and surface lobulations of the kidneys were examined. The renal vessels and loose areolar tissues from both surfaces of the pelvis were removed and cleaned. The pelvis was further traced to reach the major and minor calyces. The branching pattern of the major and minor calyces and their corresponding cap of cortical tissues were delineated.

50 fresh specimens were used for preparation of renal arterial and pelvicalyceal casts. The cast material consisted of Acrylic repair material (denture base polymer resin powder) used in dentistry, ${ }^{8}$ available in two colors, pink and clear. Pink was used for the arteries and the clear for the pelvicalyceal system. This powder was mixed with the cold cure liquid, Acrylin $\mathrm{R}$ in a ratio of 1:2; (one part powder and two parts liquid). The prepared resin was immediately injected, since it began to set in less than a minute or so.

The kidneys, along with a segment of aorta and the ureter, were removed and then washed with tap water thoroughly. The renal arteries were canulated through the aorta using an infant feeding tube. The blood from the kidneys were washed away by injecting tap water through the infant feeding tube, the washed out blood exiting via the renal vein. Once the arterial system was completely washed out and the kidneys turned pale, the cast material was prepared and then immediately injected into the canulated renal arteries, the end point being when the cast material exited via the renal vein. The renal arteries were ligated and kept aside for the material to set, approximately one hour. Following this the ureters were canulated, again with an infant feeding tube. The cast material was again freshly prepared and injected immediately. For the ureters, an amount of $6 \mathrm{ml}$ of the cast material was used, for fear of rupturing the pelvicalyceal system. After injection the ureters were also ligated and the kidneys were placed in formalin for 24 hours for the cast to set. The next day the kidneys were placed in glass jars containing concentrated sulphuric or hydrochloric acid. After a period of 24-48 hours the kidneys were removed from the acid, washed under running tap water to remove acid and any tissue debris attaching itself to the cast. Once clean, the casts were air dried and the minute branches were trimmed to observe the patterns better. The branches and the 
branching pattern of the renal artery as well as that of each segmental artery were studied. The pattern of pelvicalyceal system was also noted, along with the course of the segmental arteries with respect to the pelvicalyceal system.

Photographs were taken to document the variations. The results obtained were then tabulated.

Classification of Pelvicalyceal system?:

Multicalyceal or Radiate type:

Triangular type or Tricalyceal:

$\mathrm{Y}$ - shaped or Bicalyceal:

Presence of upper and lower major calyces with three or more minor calyces draining independently into the pelvis between the two.

Three major calyces - upper, middle, lower, formed by the fusion of the respective minor calyces open into it

The pelvis is formed by the convergence of two major calyces - the upper and lower, which may or may not be symmetrical; middle calyx is absent.

Classification of renal segmental arteries:

\begin{tabular}{|c|c|}
\hline \multicolumn{2}{|c|}{ Anterior division } \\
\hline Type I & Terminates as the US and MS arteries after giving off the LS artery. \\
\hline Type II & Terminates as the MS and LS arteries after giving off the US artery. \\
\hline Type III & Gives rise to the AS artery and then to US, MS and LS arteries. \\
\hline Type IV & $\begin{array}{l}\text { Gives off three terminal branches viz., AS, US and MS after giving off LS artery much before the } \\
\text { hilum. }\end{array}$ \\
\hline Type V & $\begin{array}{l}\text { The anterior division runs downwards, in front of the pelvis of the ureter with an outward } \\
\text { convexity from which the AS, US, MS and LS arteries arise in single or small more than one. }\end{array}$ \\
\hline Type VI & $\begin{array}{l}\text { Divides and terminates into normal } 4 \text { segments i.e. AS, US, MS and LS arteries much before the } \\
\text { hilum. }\end{array}$ \\
\hline Type VII & $\begin{array}{l}\text { Anterior division divides and terminates either in combination with two segmental arteries in } \\
\text { common i.e. apical with upper, middle with lower and apical with middle segmental arteries much } \\
\text { before the hilum or within the hilum. }\end{array}$ \\
\hline Type VIII & $\begin{array}{l}\text { Anterior segmental divisions or the branches arises directly from the aorta as accessory renal } \\
\text { artery which may replace any one or two segmental branches and supply to the definitive area } \\
\text { without anastomosing with its neighboring artery. }\end{array}$ \\
\hline \multicolumn{2}{|c|}{ Apical segment artery (AS): } \\
\hline Type I & From the anterior division of the renal artery, along with most of the other segmental arteries. \\
\hline Type II & From the upper segmental artery. \\
\hline Type III & $\begin{array}{l}\text { At the junction of the anterior and the posterior divisions of the renal artery or with the middle } \\
\text { segmental arteries. }\end{array}$ \\
\hline Type IV & From the renal artery much before its division into the other segmental arteries. \\
\hline Type V & From the aorta (superior accessory renal artery). \\
\hline Type VI & It arose from the posterior division of the renal artery. \\
\hline \multicolumn{2}{|c|}{ The types of the upper segment artery (US) } \\
\hline Type I & From the anterior division of the renal artery along with most of the other segmental arteries. \\
\hline Type II & From or along with the apical segment artery. \\
\hline Type III & From or along with the middle segmental artery. \\
\hline Type IV & From or along with the lower segmental artery. \\
\hline \multicolumn{2}{|c|}{ The types of the middle segment artery (MS): } \\
\hline Type I & From the anterior division of the renal artery along with most of the other segmental artery. \\
\hline Type II & $\begin{array}{l}\text { From or along with the upper segmental artery and divides into upper and middle within the renal } \\
\text { parenchyma or within the hilum. }\end{array}$ \\
\hline Type III & From or in common with the lower segment artery. \\
\hline Type IV & From or in common with the apical segment artery. \\
\hline Type V & $\begin{array}{l}\text { May arise directly from the aorta as an accessory renal artery either above or below the normal } \\
\text { renal artery. }\end{array}$ \\
\hline
\end{tabular}




\begin{tabular}{|l|l|}
\hline The types of the lower segment artery (LS) \\
\hline Type I & $\begin{array}{l}\text { Was seen to arise most commonly from the anterior division of the renal artery along with most of } \\
\text { the other segmental branches. }\end{array}$ \\
\hline Type II & $\begin{array}{l}\text { Arises from the posterior division of the renal artery. In these cases the posterior segmental artery } \\
\text { is comparatively larger to supply the inferior pole of the kidney. }\end{array}$ \\
\hline Type III & Given off directly from the renal artery much before the hilum. \\
\hline Type IV & Direct branch from the aorta, but along with the middle segmental artery. \\
\hline The types of posterior segment artery (PS) \\
\hline Magistral & $\begin{array}{l}\text { Curved down towards the lower pole giving off successive branches upwards, laterally, and } \\
\text { downwards. }\end{array}$ \\
\hline Bifurcating & Divided into two more or less equal-sized branches running up and down respectively. \\
\hline Cruciate & Ran straight across the back of the pelvis and calyces, giving vertical upper and lower branches. \\
\hline
\end{tabular}

\section{Results:-}

\section{Pelvicalyceal system:}

The pelvis, shows different shapes depending on the number of major calices draining into it and the angle it makes with the ureter:

\begin{tabular}{|l|c|c|c|c|}
\hline \multicolumn{2}{|c|}{ No of foetal kidneys studied by dissection } & \multicolumn{2}{l|}{ No of adult kidneys studied by dissection } \\
\hline Pattern observed & \multicolumn{2}{|c|}{ In foetal kidneys } & \multicolumn{2}{c|}{ In adult kidneys } \\
\hline & No. & $\%$ & No. & $\%$ \\
\hline Tricalyceal & 9 & $30.00 \%$ & 53 & $44.17 \%$ \\
\hline Bicalyceal & 8 & $26.67 \%$ & 29 & $24.17 \%$ \\
\hline $\begin{array}{l}\text { Multicalyceal or } \\
\text { radiate }\end{array}$ & 12 & $40.00 \%$ & 31 & $25.83 \%$ \\
\hline Others & 1 & $3.33 \%$ & 7 & $5.83 \%$ \\
\hline
\end{tabular}

'Others' included early division of the pelvis into calices, the lower calyx draining the mid-zone also, the middle calyx in line with the ureter, upper and lower calices joining it at right angles to form the pelvis, two pelvicalyceal systems (fig 1) draining separately into two ureters. In the case of double ureters, the ureter at the higher level was continuous with the upper pelvicalyceal system and the one below with the lower ureter. No cross drainage was seen between the two systems

The minor calices are oriented in the anterior and posterior planes, with respect to the frontal. This is most marked in the hilar lobe. Two or three minor calices from the anterior and posterior aspects of the kidney converge at varying angles to form the middle major calyx. In the event that the middle calyx is absent, the minor calices draining the mid zone drain either into the upper or lower major calices. The minor calices opening into the major calices can either open individually or may join to form a common stalk before joining it. 


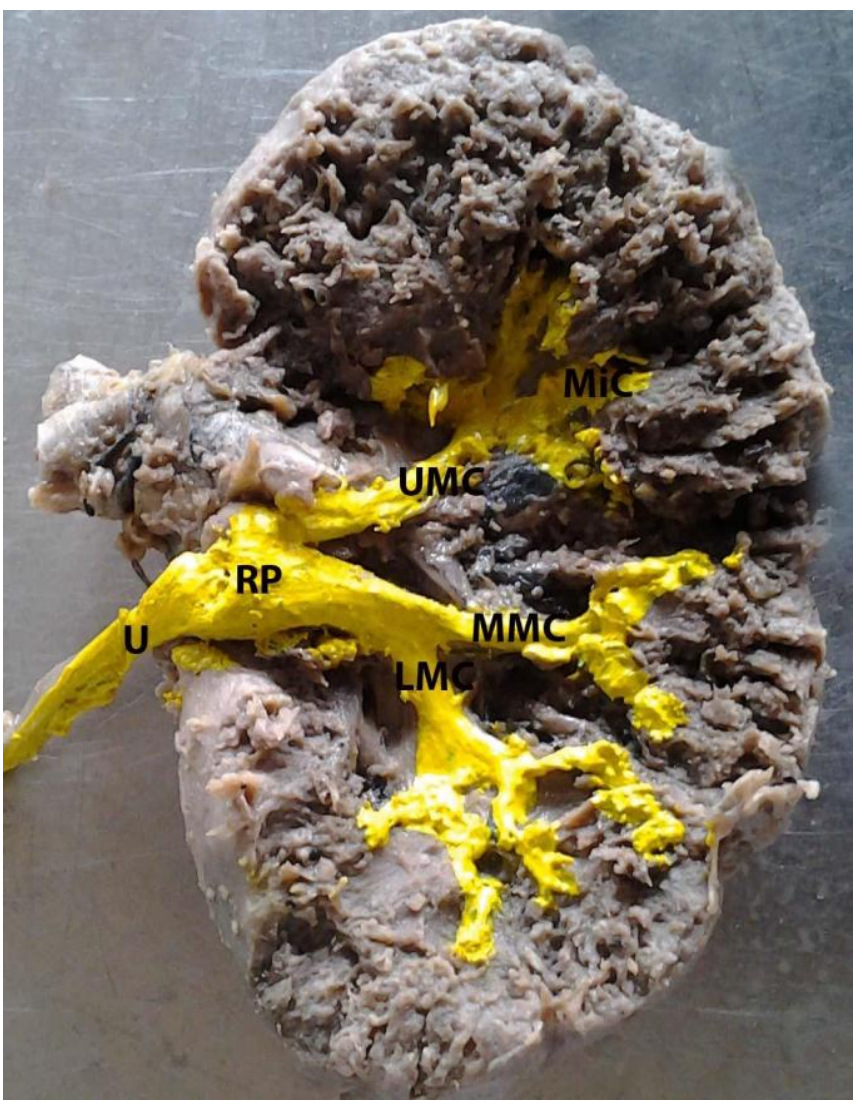

Kidney showing tricalyceal pattern with long infundibulum of UMC \& short infundibula of MMC \& LMC

RP: Renal Pelvis; UMC: Upper major calyx; MMC: Middle major calyx; LMC: Lower major calyx; MiC: Minor calyx; U: ureter

\section{Renal arterial segments:}

\begin{tabular}{|l|l|l|}
\hline \multicolumn{2}{|l|}{ Total } & $\mathbf{\%}$ \\
\hline Primary Division & 46 & $92 \%$ \\
\hline A-P & 4 & $8 \%$ \\
\hline U-L & $\mathbf{5 0}$ & $\mathbf{1 0 0 . 0 0 \%}$ \\
\hline Total & 1 & $2 \%$ \\
\hline Patterns Of Division & 22 & $44 \%$ \\
\hline TY1 & 12 & $24 \%$ \\
\hline TY2 & 2 & $4 \%$ \\
\hline TY3 & 3 & $6 \%$ \\
\hline TY4 & 2 & $4 \%$ \\
\hline TY5 & 6 & $12 \%$ \\
\hline TY6 & 2 & $4 \%$ \\
\hline TY7 & $\mathbf{5 0}$ & $\mathbf{1 0 0 . 0 0 \%}$ \\
\hline TY8 & \multicolumn{2}{l|}{} \\
\hline Total & 31 & $62 \%$ \\
\hline Variations of the upper segmental artery & 15 & $30 \%$ \\
\hline Ty1 & 3 & $6 \%$ \\
\hline Ty2 & 0 & $0 \%$ \\
\hline Ty3 & 1 & $2 \%$ \\
\hline Ty4 & $\mathbf{5 0}$ & $\mathbf{1 0 0 . 0 0 \%}$ \\
\hline other & \multicolumn{2}{l|}{} \\
\hline Total & 27 & $54 \%$ \\
\hline Variations of middle segmental artery & 8 & $16 \%$ \\
\hline Ty1 &
\end{tabular}




\begin{tabular}{|l|l|l|}
\hline Ty3 & 13 & $26 \%$ \\
\hline Ty4 & 1 & $2 \%$ \\
\hline other & 1 & $2 \%$ \\
\hline Total & $\mathbf{5 0}$ & $\mathbf{1 0 0 . 0 0 \%}$ \\
\hline Variations of the Lower Segmental Artery & \multicolumn{2}{l|}{} \\
\hline TY1 & 42 & $84 \%$ \\
\hline TY2 & 3 & $6 \%$ \\
\hline TY3 & 4 & $8 \%$ \\
\hline TY4 & 1 & $2 \%$ \\
\hline Total & $\mathbf{5 0}$ & $\mathbf{1 0 0 \%}$ \\
\hline Patterns of the posterior segmental artery & \multicolumn{2}{l|}{} \\
\hline Magistral & 31 & $62.00 \%$ \\
\hline Bifurcating & 15 & $30.00 \%$ \\
\hline Cruciate & 4 & $8.00 \%$ \\
\hline Total & $\mathbf{5 0}$ & $\mathbf{1 0 0 . 0 0 \%}$ \\
\hline
\end{tabular}
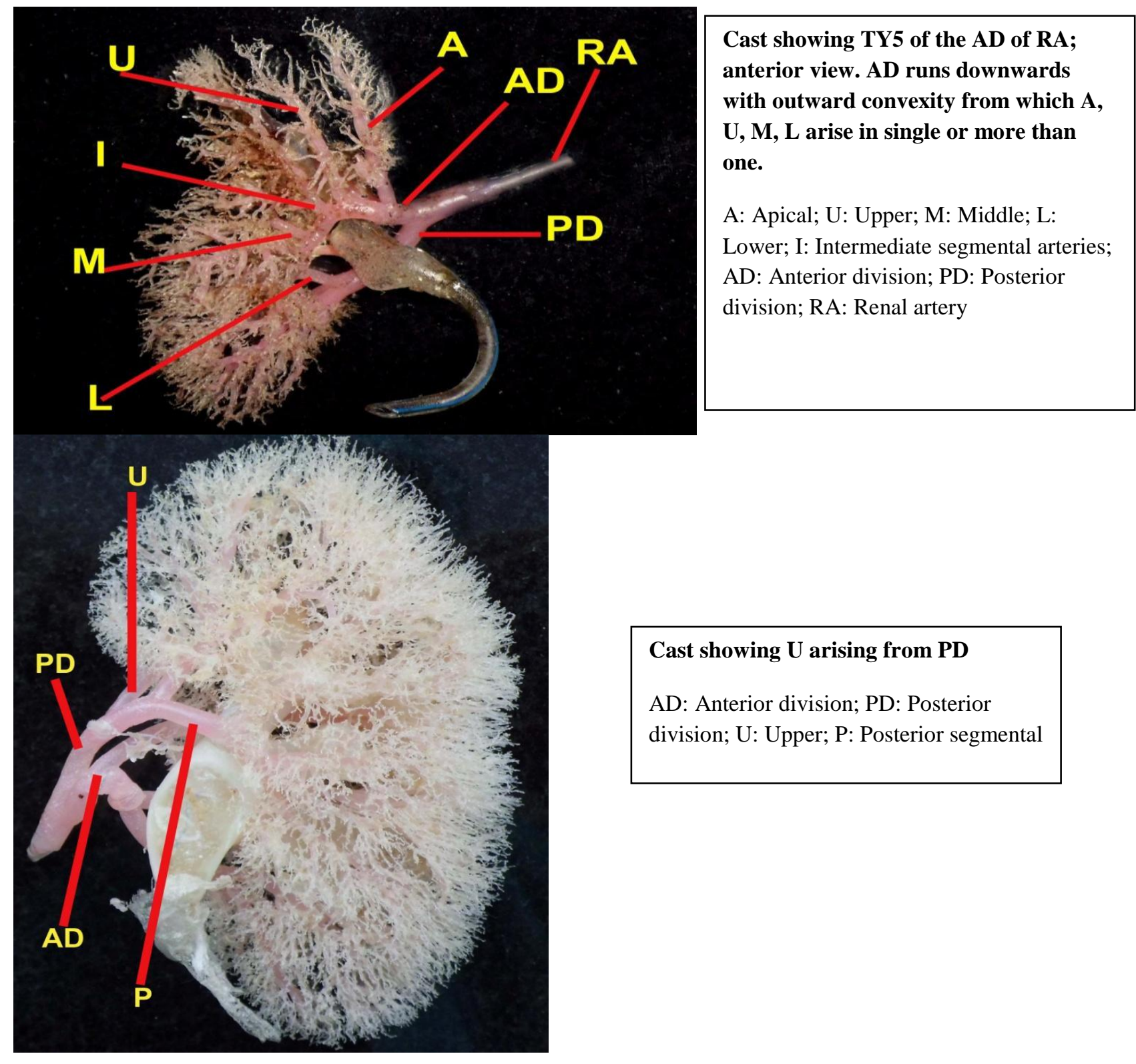

Cast showing $\mathbf{U}$ arising from $P D$

AD: Anterior division; PD: Posterior

division; U: Upper; P: Posterior segmental 


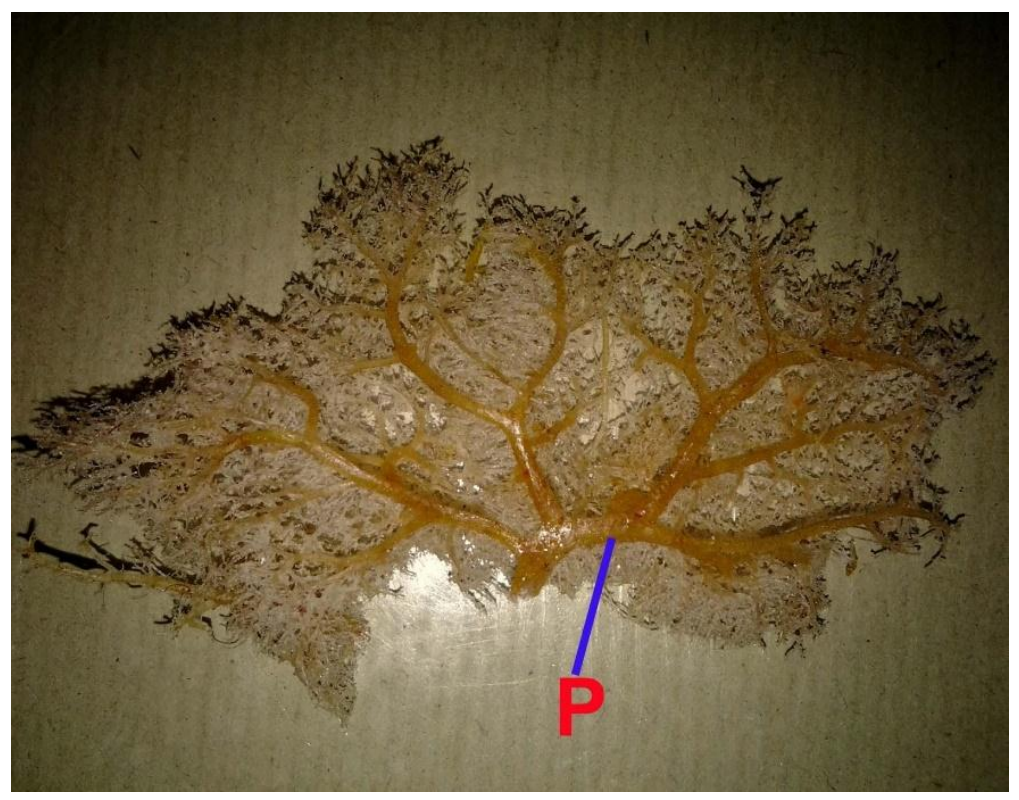

Cast showing the MAGISTRAL pattern of the posterior segmental artery. This artery was detatched from the rest of the cast.

P: Posterior segmental artery

Relationship between the segmental arteries and the renal collecting system: Superior Pole:

In the majority of the casts the arterial supply related to the upper caliceal group arose from two arteries: one originated from the anterior division and the other from the posterior division of the renal artery. In the rest, both the arteries arose from either the anterior or posterior divisions alone.

\begin{tabular}{|l|l|l|}
\hline Superior Pole Supply & Total & \% \\
\hline Anterior \& Posterior & 44 & 88 \\
\hline Anterior & 5 & 10 \\
\hline Posterior & 1 & 2 \\
\hline Total & $\mathbf{5 0}$ & $\mathbf{1 0 0}$ \\
\hline
\end{tabular}

Mid zone (Hilar):

In all cases the artery to the mid zone arose from the anterior division of the renal artery.

\section{Inferior Pole:}

The arterial supply to the front and back of the inferior pole usually arose from the inferior segmental artery. In the present study, this artery was a branch of the anterior division in $84 \%$ of the cases. The inferior segmental artery passes in front of the ureteropelvic junction and after entering the inferior pole divides into an anterior and a posterior branch. The anterior branch is related to the anterior surface and the posterior to the posterior surface of the lower infundibulum. The posterior branch progresses under the neck of the lower calyx to reach the posterior aspect of the kidney. In the rest of the casts, the anterior branch arose from the anterior division and the posterior from the posterior division.

\section{Dorsal Kidney:}

In $60 \%$ of the casts the posterior segmental artery was closely related to the upper infundibulum or to the junction of the pelvis with the upper pelvis. In the remaining casts the posterior segmental artery crossed the middle posterior surface of the renal pelvis.

\section{Discussion:-}

The present study was undertaken with an aim of better understanding the patterns of division of the pelvicaliceal system and its relation to the renal segmental arteries. 
The major calices were classified according to the simplified classification of Ninthoujam ${ }^{9}$ into tricaliceal, bicaliceal and multicaliceal, tricaliceal pattern being the most common. The proportion of multicaliceal was greater in foetal than in adult kidneys supporting the theory of fusion of calices with development.

The majority of supply to the superior pole was from the branches of the anterior division of the renal artery; the dorsal kidney from posterior division; the mid-zone was always supplied by the anterior division and the inferior pole was supplied by the inferior segmental artery in most of the cases. These findings were similar to those of Sampaio and Aragao ${ }^{7}$. However there was a single instance, where, the upper segment artery, usually arising from the anterior division or along with other segment arteries arising from the anterior division, was seen to arise from the posterior division. The present study also noted a single instance where the middle segment artery took origin from the renal artery before it divided into anterior and posterior branches. Other workers ${ }^{10}$ noted that if the caliber of the middle segment artery if small, the branch from the posterior segment artery may be larger towards its posterior segment of the kidney. This makes the middle segmental partial resection and reconstruction quite difficult for endourological surgeons ${ }^{11}$. Graves ${ }^{12,13}$ noted that in instances where the lower segment artery was a direct branch on the aorta, usually along with the middle segment artery, the gonadal artery was seen to arise from it , sometimes quite near to the kidney. Ligation of the lower segment artery must therefore be made after the artery to the gonad has originated, as otherwise testicular atrophy will eventually develop ${ }^{12,13}$. In the present study, such variations were not seen even though in $2 \%$ of casts the lower segment artery arose directly from the aorta.

In conclusion, the patterns of calices and the segmental arteries not only showed remarkable variations, but their arrangement with respect to each other also was very diverse. These innumerable combinations cannot be predicted from studying the external surface of the kidney. A surgeon should keep in mind all the possible variations and complications of each before approaching any intervention on the kidney. Needless to say, preoperative angiography and IVUs should be done as a routine before any such intervention is taken up so as to preserve and protect the healthy segments as much as possible.

\section{References:-}

1. Muhammed Shahbaz Hanif, Mubashar Husain Toori, Masood Ahmed Sheikh, Detailed calyceal anatomy for endourology, Pakistan J. Med. Res, 2004

2. David Sykes. The morphology of renal lobulations and calyces, and their relationship to partial nephrectomy. Brit. J. Surg., 1964, Vol. 51, No. 4, April.

3. Longia GS, Kumar V, Gupta CD Intrarenal arterial pattern of human kidney--corrosion cast study. Anat Anz. 1984; 155(1-5):183-94.

4. Ecaterina Dăescu, Delia Elena Zăhoi, A. Motoc, Aurora Alexa, Flavia Baderca, AlexandraEnache, Morphological variability of the renal artery branching pattern: a brief review and an anatomical study, Rom $J$ Morphol Embryol 2012, 53(2):287-291

5. Weld KJ, Bhayani SB, Belani J, Ames CD, Hruby G, Landman J, Extrarenal vascular anatomy of kidney: assessment of variations and their relevance to partial nephrectomy, Urology, 2005, 66(5):985-989.

6. Kaye, K.W., Goldberg, M.E. applied Anatomy of the kidney and ureter. Urol Clin North Am 1982; 9:3-13

7. F. J. B. Sampaio \& A. H. M. Aragao, anatomical relationship between the intrarenal arteries and the kidney collecting system; the J. Of Urology; 1989; 143:679-681.

8. J. P. De Clerck, L.S.D. Microwave polymerization of acrylic resins used in dental prostheses; J of Prosth Dentistry; 1987, 57(5):650-658.

9. Ningthoujam DD, Chongtham RD \& Sinam SS; Pelvi-Calyceal System in Foetal and Adult Human Kidneys. J. Anat. Soc. India 54 (1) 1-11 (2005)

10. Rizk ES, El-Galley and Keane TE. Embryology, anatomy, and surgical applications of the kidney and ureter. Surg Anat and Embryo. 2000; 80(1): 381-387.

11. Khamanarong K, Prachaney P, Utaravichien A, Tong-Un T, Sripaoraya K. Anatomy of renal arterial supply. Clin Anat 2004 May; 17(4): 334-336.

12. F. T Graves, 1954, the anatomy of the intrarenal arteries and its application to segmental resection of the kidney; British J of Surgery; 42, 132-139

13. Graves F. T; the aberrant renal artery. J. Anat. 1956b;90: 553-558 